the veil which Nature had drawn over her secrets. He conceived with clarity the idea of lines of force.

A series of slides, including portraits of Heuss, Euler, Young, Faraday and others, enlivened his discourse. In an enthralling way he contrasted the Nordic conception of infinity with the strange shrinking of the Semites from such infinity (the Bedouin tent). In the same spirit he explained Einstein's theory of relativity, with which he settled in great style. To the abstract mathematical junk of the Jewish physicists he opposed the living conception of high and holy laws of Nature, such as the Nordic investigator wins for himself in reverence before the logic and greatness of Nature. At the conclusion of his stirring address, Prof. Tomaschek emphasised that overloading and complicating the methods of research with many mathematical formulæ would certainly not lead to Nature. He hoped that German youth, brought once again near to this Nature, would once more find the way to meet her with clear young eyes.
Party-member Dr. A. Bühl (Karlsrühe) subjected the toaching of physics in the German schools and universities to sharp criticism. In addition to the values of humanistic education he demanded more physics even in the secondary schools, and directed attention to many questionable practices of the teachers of physics in the schools. It was much more important to provide clear and well-understood foundations than to give prominence to hypotheses, which did not acquire a meaning until the scientific struggle began.

Geheimrat Lenard delivered the concluding words, and expressed his approval of these addresses. $\mathrm{He}$ exhorted all to continue energetically the fight against the Jewish spirit, which had by no means vanished from the German universities. He recounted many examples of Jewish arrogance (Einstein), supported by Jewish publishing houses (Springer), and expressed his confidence that this movement for German co-operation would embrace all our centres of higher learning.

\title{
Synthesis of a Natural Colouring Matter
}

$\mathrm{O}^{\mathrm{R}}$ RGANIC chemists have devoted much time and ingenuity to building up the complex products of life ever since Wöhler and liebig first broke down the barrier which had separated them from the artificial products of the laboratory, by their synthesis of urea. Sometimes the problems presented are of a peculiarly baffling nature, and we can only marvel at the astonishing progress which has been made both in unravelling and in reassembling the intricate structures of molecular architecture found in Nature. The prolonged and brilliant researches of Emil Fischer upon sugars, proteins and purines have inspired later workers to engage upon still more elusive problems. In Great Britain, Prof. R. Robinson, of Oxford, has already succeeded in laying bare the constitution of numerous alkaloids, colours and other products of plant life. Among these are the anthocyanins or the pigments of flowers. The rapid growth of this branch of chemistry in recent years is apt to become bewildering to the student. We are therefore indebted to Prof. Robinson for the clear exposition which he gave in his Friday evening discourse on November 15 at the Royal Institution, describing the general lines on which one single investigation was carried out, with the view of illustrating the special technique which has had to be evolved.

The pigment chosen was that of the scarlet Pelargonium, which occurs also in pink carnations and red dahlias. The problem was approached by three separate methods, namely, isolation as a chemical individual and characterisation of the purified natural pigment, analytical dismemberment of the complex molecule into simpler ones of known constitution, and finally, synthesis therefrom of the identical compound by methods which leave no doubt as to the constitution. The analytical and synthetical methods are complementary and entirely independent of one another. Proof of structure depends equally on both.

Extraction of the pigment from petals by solvents was effected in 1903 by Griffiths and in 1905 by Molisch. In 1911 Grafe, using Molisch's method, extracted $10 \mathrm{gm}$. of a beautiful crystalline pigment from $28 \mathrm{kgm}$. of fresh petals. A greatly improved method was devised by Willstätter and Bolton in 1915, who obtained pelargonin chloride, $\mathrm{C}_{27} \mathrm{H}_{31} \mathrm{O}_{15} \mathrm{Cl}_{1} 4 \mathrm{H}_{2} \mathrm{O}$, although the natural colour is probably the tartrate. The chloride can be prepared from the natural pigment, and is more suitable for characterisation than the tartrate. Hydrolysis by acids splits the molecule of this chloride into pelargonidin chloride, $\mathrm{C}_{15} \mathrm{H}_{11} \mathrm{O}_{5} \mathrm{Cl}$, and glucose, the reaction being

$$
\mathrm{C}_{27} \mathrm{H}_{31} \mathrm{O}_{15} \mathrm{Cl}+2 \mathrm{H}_{2} \mathrm{O}=\mathrm{C}_{15} \mathrm{H}_{11} \mathrm{O}_{5} \mathrm{Cl}+2 \mathrm{C}_{6} \mathrm{H}_{12} \mathrm{O}_{6} \text {. }
$$

Removal of glucose in this way has no marked effect on the colour, so that the chromophore has evidently not been broken down. Thus pelargonin chloride appears to be a diglucoside of pelargonidin chloride. By partial hydrolysis it has also been possible to obtain a monoglucoside, and it was 
observed that both glucosides were strongly fluorescent substances. It was, however, necessary to determine the mode of linking of the glucose to the pelargonidin. Now glucose occurs most frequently in the form of $\beta$-glucosides, in which the hydroxyl groups lie alternately above and below the plane of the pyranose ring. This gave a clue to the probable structure which afterwards proved to be correct; but it was also necessary to ascertain whether the glucose units were linked together or to different points of the main structure. The latter formation was ultimately adopted.

Further degradation of the compound by means of fusion with alkali yielded the well-known substances phloroglucinol and $p$-hydroxybenzoic acid. We may therefore infer that these two aromatic compounds have been condensed together in such a way as to form a pyrylium ring, since salt formation shows that we are dealing with an oxonium compound.

This limits the choice to two structures only, namely :

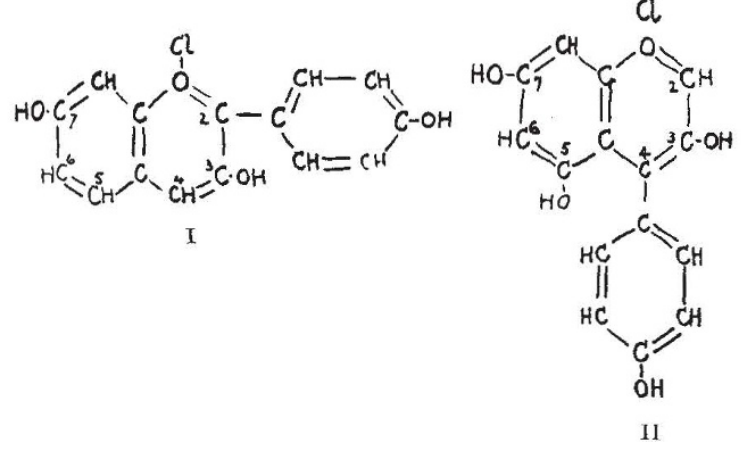

The reasons for this limitation are as follows: (i) The results of fusion show that one hydroxyl group must be in the pyrylium ring and (ii) the latter would be unstable if the hydroxyl group were at positions 2 or 4 . The final choice depends on synthetic methods.

The location of the two glucose units was then determined by careful study of the properties of numerous synthetic pelargonium derivatives of known constitution. In this way it was discovered that the presence of unsubstituted hydroxyl groups in certain positions exerts a marked influence upon the physical properties of the compounds. Thus the strong fluorescence of the glucosides indicates the protection of the hydroxyl group at position 5 by glucose, whilst the stability of the pigment in the presence of weak acids containing traces of iron is evidence of similar protection at position 3 . Hence pelargonin is probably a 3.5 diglucoside. This argument involved a vast amount of experimental work, but it certainly did make possible the prediction of the structure of pelargonin. This was finally confirmed by synihesis.

No further deductions could be drawn from analytical data, and it became necessary to attempt the synthesis of the pigment. Now it is known that pyrylium derivatives result from the condensation of $o$-hydroxybenzaldehydes with compounds containing the group $-\mathrm{CO}-\mathrm{CH}_{2}-$ in the presence of hydrogen chloride. But before attempting this condensation, it was necessary to introduce the glucose groups (protected by acetylation) into the two reacting compounds at the positions already diagnosed. This was accomplished by means of tetracetylglucosidylbromide, the required components being acetylated monoglucosides of phloroglucinaldehyde (III) and $\omega .4$ dihydroxyacetophenone (IV).

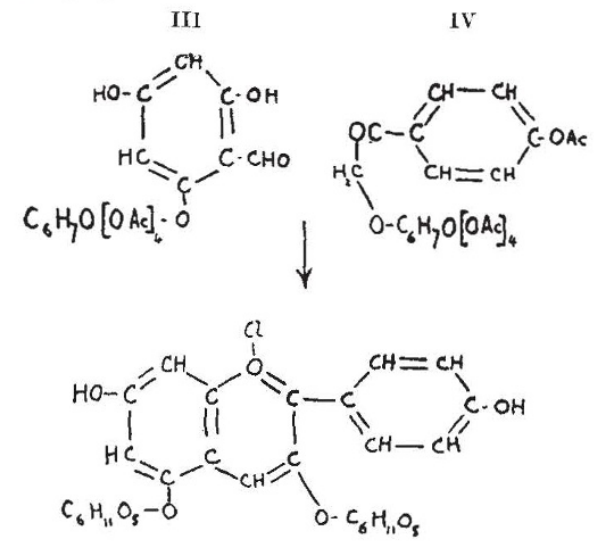

The constitution of the former was proved by methylating the two free hydroxyl groups, hydrolysing the resulting dimethoxyglucoside to a dimethylether of phloroglucinaldehyde and proving that the latter would still undergo the pyrylium condensation. This fixed the position of the glucose molecule with certainty. The constitution of the other compound follows from its synthesis from $p$-acetoxybenzoylchloride and diazomethane to $p$-acetoxydiazoacetophenone, which on careful hydrolysis gave the corresponding carbinol. From this the required acetylated glucoside was prepared.

Condensation of these two products in the cold with hydrogen chloride yielded a pyrylium salt (v) which can have only one constitution. When the acetyl groups were finally removed and the resulting compound was carefully purified, it was found to be identical with pelargonin chloride, which is therefore the chloride of pelargonidin-3-5 di $\beta$-glucoside. Comparison with the natural pigment was made by a detailed examination of many of its physical properties, no divergences having been found. 